\section{Estudo da sobrevida de pacientes com câncer de mama atendidas no hospital da Universidade Federal de Santa Maria, Rio Grande do Sul, Brasil}

\author{
Survival study of breast cancer patients \\ treated at the hospital of the Federal University \\ in Santa Maria, Rio Grande do Sul, Brazil
}

Anaelena Bragança de Moraes 1,2 Roselaine Ruviaro Zanini 1,2 Marcelo Scalvenzi Turchiello 1 João Riboldi 2

Lídia Rosi de Medeiros 2

\author{
1 Departamento de \\ Estatística, Universidade \\ Federal de Santa Maria, \\ Santa Maria, Brasil. \\ 2 Programa de Pós-graduação \\ em Epidemiologia, \\ Universidade Federal \\ do Rio Grande do Sul, \\ Porto Alegre, Brasil. \\ Correspondência \\ A. B. Moraes \\ Departamento de Estatística, \\ Universidade Federal \\ de Santa Maria. \\ Faixa de Camobi $\mathrm{km} 9$ \\ CCNE-Prédio 13, \\ Santa Maria, RS \\ 97105-900, Brasil. \\ anaelena@smail.ufsm.br
}

\section{Abstract}

This retrospective hospital-based study aimed to describe health conditions and to estimate the survival of 252 patients diagnosed with breast cancer and treated at the Mastology Outpatient Clinic at the University Hospital of the Federal University in Santa Maria, Rio Grande do Sul, Brazil, from 1980 to 2000. Analysis followed the Kaplan-Meier and Cox model. Mean age was 54 , and $73.4 \%$ of the patients had a histological diagnosis of invasive ductal carcinoma, $63.9 \%$ showed no lymph node involvement, and 57.6\% were clinical stage II. At the end of the study, 64.7\% were alive and free of breast cancer and $5.1 \%$ had died of other causes. Fiveyear survival was $87.7 \%$ for all women, and prognostic factors associated with survival were tumor size (HR = 12.03; > 5cm), lymph node involvement $(H R=3.08 ; \mathrm{N1})$ and number $(H R=4.66$; None $)$, and estrogen $(H R=0.34)$ and c-erbB-2 $(H R=2.51)$ receptors. Based on the results, intensive awareness-raising campaigns are vitally important for implementing breast cancer screening to achieve early diagnosis.

Survival Analysis; Breast Neoplasms; Delivery of Health Care; Women's Health

\section{Introdução}

O câncer de mama é o mais freqüente, sendo responsável por cerca de $10 \%$ de todos os tipos de câncer no mundo. A incidência mundial vem crescendo nesses últimos anos passando de 572 mil em 1980 para 1.050 .000 em 2000, sendo responsável por um total de 370 mil mortes no mundo no ano de 2000 1. Entretanto, existem diferenças geográficas na distribuição, havendo maior incidência nos países ocidentais e industrializados em comparação com países da África e da Ásia 1. No Brasil, o câncer de mama é a neoplasia maligna de maior incidência entre as mulheres, sendo responsável por $15 \%$ do total de mortes por câncer. Foi estimado, para o ano de 2005, o diagnóstico de 49.470 novos casos de câncer de mama 2 .

O câncer de mama, quando diagnosticado em fases iniciais, tem grandes chances de cura, com uma sobrevida de $97 \%$ em cinco anos 3 . A sobrevida é o parâmetro mais utilizado para avaliar resultados na área oncológica, inclusive epidemiológica, onde as taxas de mortalidade em séries históricas são de alta relevância analítica, sendo possível abordar técnicas estatísticas de análise de sobrevida com observações obtidas em registros de serviços de saúde. Alguns fatores prognósticos para a sobrevida global em câncer de mama são: o tamanho do tumor, o status dos linfonodos e dos receptores hormonais, o grau histológico e a idade 4 . 
Portanto, o principal objetivo deste estudo retrospectivo de base hospitalar foi descrever as principais características relacionadas à neoplasia de mama e estimar a sobrevida das pacientes, de acordo com as co-variáveis disponíveis nos registros do Hospital Universitário de Santa Maria (HUSM), que se destaca como referência na prestação de serviços públicos e gratuitos em saúde, na região central do Estado do Rio Grande do Sul, Brasil.

\section{Método}

A população deste estudo é composta por uma coorte retrospectiva de base hospitalar de mulheres, com diagnóstico prévio de câncer de mama, que consultaram no Ambulatório de Mastologia do HUSM no período de 1980 a 2000, residentes na cidade de Santa Maria e provenientes de outras localidades da região central do Rio Grande do Sul.

As variáveis estudadas foram obtidas pela revisão dos prontuários e atualização dos registros clínicos e patológicos das pacientes, os quais permitiram a elaboração de um banco de dados composto por 286 pacientes do sexo feminino. Destas pacientes, 34 foram excluídas devido à falta de dados no prontuário ou porque não retornaram mais ao ambulatório após o diagnóstico do câncer, totalizando 252 pacientes.

O tempo de sobrevida, em meses, foi definido como o período entre a data da cirurgia (obtida dos registros médicos) e a ocorrência de óbito ou da última consulta. O evento de interesse foi o óbito atribuído apenas ao câncer de mama, segundo a declaração de óbito. No final do estudo, todas as pacientes que não tinham o registro do evento, porque estavam vivas, porque não foram acompanhadas até o final ou porque morreram por outras causas, contribuíram com o seu tempo de acompanhamento na composição do grupo de risco, tendo essas pacientes recebido o registro do evento como censura.

Foram investigadas variáveis sociodemográficas (baseadas em informações registradas na primeira consulta) e clínicas, incluindo: idade, raça, tipo de tratamento (cirurgia, radioterapia, quimioterapia), detalhes da patologia do tumor (tamanho do tumor, tipo e grau histológico), comprometimento dos linfonodos regionais e número de linfonodos ressecados, presença de metástase, receptores hormonais e c-erbB- 2 .

As informações sobre a idade foram agrupadas em três faixas (40 anos ou menos, 41 a 50 anos e 51 ou mais anos). O tipo histológico do tumor foi classificado em: lobular invasivo, inflamatório, ductal invasivo ou outros. Para o estadiamento clínico do tumor foi utilizada a classificação TNM ( $\mathrm{T}$ = tumor, $\mathrm{N}=$ linfonodo, $\mathrm{M}=$ metástase) 5. O tamanho do tumor foi agrupado em três faixas: menor ou igual a $2 \mathrm{~cm}$ (T1), 2 a $5 \mathrm{~cm}$ (T2) e maior que $5 \mathrm{~cm}$ (T3). O comprometimento dos linfonodos foi classificado em: N0 (sem comprometimento dos linfonodos), N1 (metástase em linfonodos axilares homo-laterais móveis) e N2 (metástase em linfonodos axilares fixos uns aos outros ou a outras estruturas). A metástase foi classificada em: M0 (ausência de metástase à distância) e M1 (presença de metástase à distância) no diagnóstico da doença 5 .

O número de linfonodos comprometidos e ressecados foi categorizado, respectivamente, em: nenhum, 1 a 3 ou maior que 3 e nenhum, 1 a 10 ou maior que 10 . Foi utilizado o grau histológico combinado de Nottingham, classificado em: bem diferenciado (I), moderadamente diferenciado (II) e indiferenciado (III) 6 .

Foi realizada uma análise descritiva de todas as co-variáveis.

Para estimar as probabilidades de sobrevida a cada tempo foi utilizado o estimador de Kaplan-Meier, sem e com estratificação. Para as comparações entre as diversas curvas foi utilizado o teste de log-rank, dada a proporcionalidade dos riscos.

Com o objetivo de estimar os efeitos de cada co-variável na sobrevida das pacientes, ajustou-se modelos de Cox para cada uma delas e a partir da significância dos riscos relativos a cada tempo (definidos pela exponencial dos parâmetros), definiu-se quais as que entrariam no modelo múltivariável 7 .

Para a modelagem da função de sobrevida foi utilizado o modelo de riscos proporcionais de Cox ${ }^{8}$. Na análise multivariável inicial, todas as variáveis foram incluídas no modelo, sendo retiradas e recolocadas posteriormente, uma de cada vez, segundo o nível de significância estatístico de 0,05 , estabelecido para os coeficientes, conforme resultado do teste de Wald e do teste da razão da verossimilhança entre os modelos ajustados. Além disso, foram testadas todas as possíveis interações entre variáveis.

As variáveis que permaneceram no modelo final foram as que se mostraram estatística e epidemiologicamente significantes, depois de controladas pelas demais no modelo de Cox múltivariável. O pressuposto da proporcionalidade necessária ao modelo de Cox foi testado com o método gráfico e com o teste da co-variável dependente no tempo ${ }^{8}$.

Todas as análises foram realizadas com o programa estatístico SPSS 10.0 (SPSS Inc., Chi- 
cago, Estados Unidos) e Statistica 5.0 para Windows (Statsoft Inc.; http://www.statsoft.com).

Os registros das pacientes foram obtidos junto ao Ambulatório de Mastologia do Serviço de Ginecologia e Obstetrícia do HUSM, sem a identificação das mesmas, atendendo aos critérios da Resolução 196/96 do Conselho Nacional de Saúde.

\section{Resultados}

Os resultados foram obtidos da análise de uma amostra de 252 pacientes atendidas no Ambulatório de Mastologia do HUSM, no período de 1980 a 2000. A maioria das pacientes 160 (63,5\%) era proveniente de cidades da região central do Estado do Rio Grande do Sul, sendo que 92 $(36,5 \%)$ eram residentes em Santa Maria. Das 252 pacientes, 136 (54\%) eram procedentes dos serviços do Hospital Universitário de Santa Maria, enquanto 116 (46\%) provieram de outro serviço.

Na Tabela 1 são apresentadas as características das principais co-variáveis do estudo. A idade média das pacientes foi de 54,0 anos (amplitude 21 a 89 anos), sendo que 236 (93,6\%) eram de raça branca. Deve-se salientar que $55,1 \%$ das pacientes submetidas à cirurgia tinham idade igual ou acima de 51 anos e somente $16,3 \%$ tinham idade inferior a 40 anos. Entretanto, a idade não mostrou significância estatística na análise de sobrevida, muito provavelmente pelo pequeno número de pacientes com idade inferior a 40 anos na amostra.

Os tipos histológicos encontrados foram: ductal invasivo $(73,4 \%)$, lobular invasivo $(6 \%)$, inflamatório (8,3\%) e outros (12,3\%). Quanto ao grau histológico, evidenciou-se ser bem diferenciado $(38,8 \%)$, moderadamente diferenciado $(51,6 \%)$ e indiferenciado $(9,6 \%)$. Considerando a classificação TNM, 131 pacientes $(52 \%)$ apresentaram tamanho do tumor entre 2 e $5 \mathrm{~cm}$ (T1) e 60 pacientes $(23,8 \%)$ apresentaram tamanho do tumor acima de $5 \mathrm{~cm}$ (T3), sendo 19 (7,5\%) com presença de metástase. Entretanto, 152 pacientes $(60,3 \%)$ não apresentaram linfonodos comprometidos.

O número médio e o mediano de linfonodos ressecados foram 14,7 e 16, respectivamente, enquanto que o número médio de linfonodos comprometidos foi 2,5 e a mediana zero. A maioria das pacientes $170(67,5 \%)$ apresentou mais de 10 linfonodos ressecados.

O estadiamento clínico para as pacientes que foram a óbito foi: I - 1 (2,6\%) paciente, IIa $7(18,4 \%)$ pacientes, IIb - $12(31,6 \%)$ pacientes, IIIa+IIIb - $9(23,7 \%)$ pacientes e IV - $9(23,7 \%)$ pacientes. As pacientes com estadiamento clínico acima de IIa apresentaram taxa de óbito significativa (Tabela 1).

O tempo mediano de seguimento das pacientes foi de 62 meses (amplitude 0,4 a 241 meses). O número total de óbitos, por câncer de mama, no final do período de estudo, foi de $38(15,1 \%)$ pacientes. A média da idade destas pacientes que faleceram era 51,8 anos (amplitude 27 a 89 anos).

Ao final do estudo, 163 (64,7\%) das pacientes estavam vivas e sem câncer de mama, 38 $(15,1 \%)$ estavam vivas e com recidiva da doença, 38 (15,1\%) morreram devido ao câncer de mama e 13 (5,1\%) morreram por outra causa.

A sobrevida global obtida pela estimativa de Kaplan-Meier foi de $68,1 \%$ ao final do período de estudo, enquanto que em 5 e 10 anos a sobrevida estimada foi de $87,7 \%$ e $78,7 \%$, respectivamente, conforme Figura 1.

As probabilidades de sobrevida em cinco anos para as pacientes nos estágios I, IIa, IIb, IIIa+IIIb e IV foram 97\%, 96\%, 90\%, 73\% e 57\% e, em dez anos, foram 97\%, 87\%, 70\% 73\% e $0 \%$, respectivamente.

Na Tabela 2 são apresentados o número e o percentual de casos e óbitos, por tipo de tratamento realizado pelas pacientes. A mastectomia tipo Patey foi o procedimento adotado na maioria dos casos 152 (60,3\%), estando associada ou não aos outros tratamentos, conforme avaliação médica. Quando fizeram essa cirurgia, os marcadores foram: $\mathrm{RE}-13(34,2 \%)$ negativo e $18(47,4 \%)$ positivo; RP - $3(7,9 \%)$ negativo e $6(15,7 \%)$ positivo; p53 - $16(42,1 \%)$ negativo e 12 (31,6\%) positivo; ki67 - $1(2,6 \%)$ negativo e 25 (65,8\%) positivo; c-erbB-2 - $17(44,7 \%)$ negativo e $12(31,6 \%)$ positivo. Dessas, $62,5 \%$ foram submetidas à quimioterapia adjuvante e $61,8 \%$ à radioterapia pós-cirúrgica.

Em relação aos marcadores tumorais, 171 $(67,8 \%)$ pacientes realizaram teste para receptor de estrógeno, 39 (15,5\%) para progesterona, $140(55,5 \%)$ para p53, 131 (52\%) para ki67 e 145 $(57,5 \%)$ para c-erbB- 2 .

Das pacientes que realizaram testes de marcadores tumorais, $119(69,6 \%)$ apresentaram receptor de estrógeno, $21(53,8 \%)$ de progesterona, $53(37,9 \%)$ p53, $121(92,4 \%)$ ki67 e 51 $(35,2 \%)$ c-erbB-2.

Os resultados do modelo de regressão de Cox são apresentados na Tabela 3, que mostrou que as co-variáveis: tamanho do tumor, comprometimento dos linfonodos regionais, número de linfonodos ressecados e marcadores tumorais: receptor de estrógeno e c-erbB-2 afetam, significativamente, a sobrevida das pacientes com câncer de mama $(\mathrm{p}<0,05)$. 
Distribuição das pacientes com câncer de mama segundo as co-variáveis do estudo.

\begin{tabular}{|c|c|c|c|c|c|c|}
\hline \multirow[t]{2}{*}{ Co-variáveis } & \multicolumn{2}{|c|}{ Casos } & \multicolumn{2}{|c|}{ Óbitos* } & \multirow[t]{2}{*}{$\mathbf{p}^{\star \star}$} & \multirow{2}{*}{$\begin{array}{l}\text { Risco relativo }{ }^{\star \star \star} \\
\text { (IC } 95 \%)\end{array}$} \\
\hline & $\mathrm{n}$ & $\%$ & $\mathrm{n}$ & $\%$ & & \\
\hline \multicolumn{7}{|l|}{ Idade (anos) } \\
\hline$\leq 40$ & 41 & 16,3 & 8 & 19,5 & & 1,00 \\
\hline $41-50$ & 72 & 28,6 & 11 & 15,3 & & $0,98(0,39-2,44)$ \\
\hline$\geq 51$ & 139 & 55,1 & 19 & 13,7 & 0,84 & $0,92(0,40-2,12)$ \\
\hline \multicolumn{7}{|l|}{ Tipo histológico } \\
\hline Outros & 31 & 12,3 & 2 & 6,5 & & 1,00 \\
\hline Ductal invasivo & 185 & 73,4 & 26 & 14,1 & & $1,86(0,44-7,87)$ \\
\hline Inflamatório & 21 & 8,3 & 5 & 23,8 & & $4,12(0,80-21,28)$ \\
\hline Lobular invasivo & 15 & 6,0 & 5 & 33,3 & 0,00 & $5,70(1,11-29,45)$ \\
\hline \multicolumn{7}{|l|}{ Grau histológico\# } \\
\hline 1 & 73 & 38,8 & 11 & 15,1 & & 1,00 \\
\hline II & 97 & 51,6 & 12 & 12,4 & & $0,75(0,33-1,69)$ \\
\hline III & 18 & 9,6 & 1 & 5,6 & 0,50 & $0,34(0,04-2,64)$ \\
\hline \multicolumn{7}{|c|}{$\begin{array}{l}\text { Número de linfonodos } \\
\text { comprometidos\# }\end{array}$} \\
\hline Nenhum & 152 & 60,3 & 23 & 15,1 & & 1,00 \\
\hline $1-3$ & 48 & 19,1 & 5 & 10,4 & & $0,67(0,25-1,76)$ \\
\hline$>3$ & 51 & 20,3 & 10 & 19,6 & 0,67 & $1,29(0,61-2,70)$ \\
\hline \multicolumn{7}{|c|}{$\begin{array}{l}\text { Número de linfonodos } \\
\text { ressecados } \#\end{array}$} \\
\hline$>10$ & 170 & 67,5 & 15 & 8,8 & & 1,00 \\
\hline $1-10$ & 31 & 12,3 & 6 & 19,4 & & $2,22(0,86-5,72)$ \\
\hline Nenhum & 50 & 19,8 & 17 & 34,0 & 0,00 & $5,00(2,49-10,03)$ \\
\hline \multicolumn{7}{|c|}{ Tamanho do tumor $(\mathrm{cm})$} \\
\hline $\mathrm{T} 1(\leq 2)$ & 61 & 24,2 & 2 & 3,3 & & 1,00 \\
\hline T2 (2-5) & 131 & 52,0 & 21 & 16,0 & & $4,53(1,06-19,32)$ \\
\hline $\mathrm{T} 3(>5)$ & 60 & 23,8 & 15 & 25,0 & 0,00 & $8,06(1,84-35,25)$ \\
\hline \multicolumn{7}{|c|}{$\begin{array}{l}\text { Linfonodos regionais } \\
\text { comprometidos }\end{array}$} \\
\hline NO & 161 & 63,9 & 16 & 9,9 & & 1,00 \\
\hline N1 & 58 & 23,0 & 13 & 22,4 & & $2,36(1,13-4,92)$ \\
\hline N2 & 32 & 12,7 & 8 & 25,0 & 0,01 & $3,30(1,41-7,74)$ \\
\hline \multicolumn{7}{|l|}{ Metástase } \\
\hline MO & 233 & 92,5 & 29 & 12,4 & & 1,00 \\
\hline M1 & 19 & 7,5 & 9 & 47,4 & 0,00 & $8,52(3,84-18,87)$ \\
\hline \multicolumn{7}{|c|}{ Estadiamento clínico } \\
\hline 1 & 50 & 19,8 & 1 & 2,6 & & 1,00 \\
\hline $11 a$ & 87 & 34,6 & 7 & 18,4 & & $3,51(0,43-28,55)$ \\
\hline $\mathrm{Ilb}$ & 58 & 23,0 & 12 & 31,6 & & $9,41(1,22-72,53)$ \\
\hline IIla e IIIb & 38 & 15,1 & 9 & 23,7 & & $12,64(1,60-99,98)$ \\
\hline IV & 19 & 7,5 & 9 & 23,7 & 0,00 & $48,47(6,06-387,52)$ \\
\hline
\end{tabular}

* Número e percentual de indivíduos que morreram no período de estudo:

** Teste log-rank para a comparação das curvas de sobrevida de Kaplan-Meier:

*** Risco relativo obtido pelo uso do modelo de riscos proporcionais de Cox univariado;

\# O total (n) de cada co-variável difere devido à ocorrência de dados faltantes. 
Figura 1

Curva de sobrevida (Kaplan-Meyer) das pacientes do estudo, após a cirurgia.

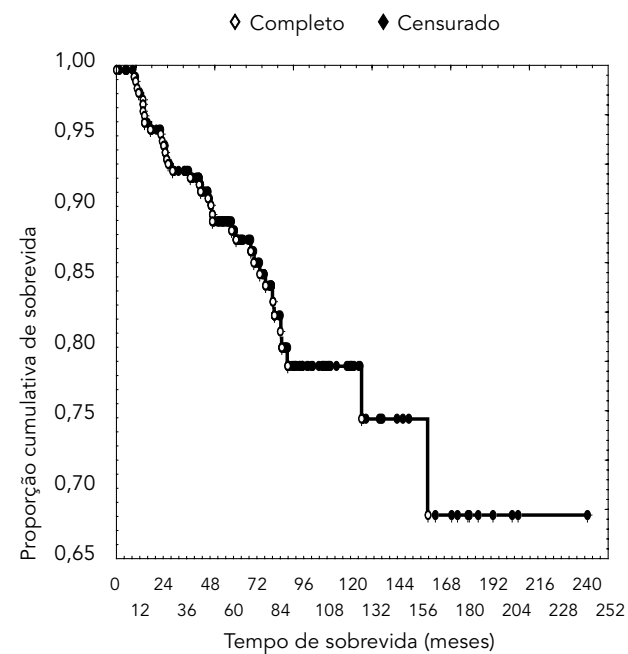

Na Figura 2 são mostradas as curvas de sobrevida estratificadas para as co-variáveis que permaneceram no modelo de regressão de Cox.

As curvas para o tamanho do tumor mostraram a existência de grande diferença na distribuição dos tempos de sobrevida entre as pacientes que tinham o tumor menor ou igual a $2 \mathrm{~cm}$, em relação as que apresentavam tumor maior do que $2 \mathrm{~cm}$. Observou-se uma diferenciação mais acentuada nas três curvas a partir de 84 meses ( 7 anos) de sobrevida.

Em relação ao número de linfonodos ressecados, observou-se que a sobrevida das mulheres do estrato que não apresentou nenhum linfonodo ressecado foi bem menor do que a dos outros dois estratos, principalmente a partir de 24 meses (2 anos) de sobrevida.

As curvas de sobrevida da co-variável linfonodos regionais comprometidos mostraram diferença significativa acentuada, desde o início do acompanhamento, sendo maior a sobrevida das pacientes sem comprometimento dos linfonodos (N0).

Tabela 2

Distribuição das pacientes com câncer de mama segundo o tratamento administrado.

\begin{tabular}{|c|c|c|c|c|c|c|}
\hline \multirow[t]{2}{*}{ Co-variáveis } & \multicolumn{2}{|c|}{ Casos } & \multicolumn{2}{|c|}{ Óbitos* } & \multirow[t]{2}{*}{$p^{\star \star}$} & \multirow{2}{*}{$\begin{array}{l}\text { Risco relativo } \\
\text { (IC } 95 \%)\end{array}$} \\
\hline & $\mathrm{n}$ & $\%$ & $\mathrm{n}$ & $\%$ & & \\
\hline \multicolumn{7}{|l|}{ Tratamento cirúrgico } \\
\hline Outros & 83 & 33,0 & 13 & 15,7 & & 1,00 \\
\hline Mastectomia total (Patey) & 152 & 60,3 & 15 & 9,9 & & $0,46(0,22-0,98)$ \\
\hline Não realizou & 17 & 6,7 & 10 & 58,8 & 0,03 & $7,40(3,20-17,12)$ \\
\hline \multicolumn{7}{|l|}{ Quimioterapia } \\
\hline Primária & 19 & 7,5 & 10 & 52,6 & & 1,00 \\
\hline Adjuvante & 141 & 56,0 & 20 & 14,2 & & $0,09(0,04-0,20)$ \\
\hline Neo-adjuvante & 20 & 8,0 & 3 & 15,0 & & $0,12(0,03-0,45)$ \\
\hline Não realizou & 72 & 28,5 & 5 & 6,9 & 0,00 & $0,03(0,01-0,10)$ \\
\hline \multicolumn{7}{|l|}{ Radioterapia pós-operatória } \\
\hline Não & 99 & 39,3 & 18 & 18,2 & & 1,00 \\
\hline Sim & 153 & 60,7 & 20 & 13,1 & 0,12 & $0,61(0,32-1,15)$ \\
\hline \multicolumn{7}{|l|}{ Radioterapia pré-operatória } \\
\hline Não & 244 & 96,8 & 37 & 15,2 & & 1,00 \\
\hline Sim & 8 & 3,2 & 1 & 12,5 & 0,95 & $0,94(0,13-6,92)$ \\
\hline
\end{tabular}

* Número e percentual de indivíduos que morreram no período de estudo;

** Teste log-rank para a comparação das curvas de sobrevida de Kaplan-Meier;

*** Risco relativo obtido pelo uso do modelo de riscos proporcionais de Cox univariado. 
Tabela 3

Modelo multivariável para as pacientes com câncer de mama atendidas no Ambulatório de Mastologia do Hospital Universitário de Santa Maria, de 1980 a 2000.

\begin{tabular}{lc}
\hline Co-variáveis & Risco relativo* (IC95\%) \\
\hline $\begin{array}{l}\text { Tamanho do tumor (cm) } \\
\leq 2\end{array}$ & 1,00 \\
$2-5$ & $4,01(0,50-32,16)$ \\
$>5$ & $12,03(1,44-100,48)$ \\
Linfonodos regionais comprometidos & 1,00 \\
N0 & \\
N1 & $3,08(1,24-7,66)$ \\
N2 & $2,31(0,77-6,88)$ \\
Número de linfonodos ressecados & 1,00 \\
$>10$ & $2,17(0,62-7,57)$ \\
1 a 10 & $4,66(1,81-12,02)$ \\
Nenhum & \\
Receptor de estrógeno & $0,34(0,14-0,83)$ \\
c-erbB-2 & $2,51(1,01-6,27)$ \\
\hline
\end{tabular}

* Risco relativo obtido pelo uso do modelo de riscos proporcionais de Cox multivariável. te hospitalar de pacientes do sexo feminino do Rio de Janeiro (80\%), com câncer de mama invasor, e inferior à encontrada por Miguel et al. 12, em um estudo de base hospitalar realizado em Cuba (95,4\%).

A sobrevida em cinco anos, para as pacientes no estágio I, foi de $97 \%$, similar à encontrada por Vázquez et al. 13 e Miguel et al. 12, e superior à obtida por Mora-Díaz \& Sánchez-Redonet 14. Para pacientes no estágio IIa e IIb a sobrevida (96\%) foi superior a outros estudos 12,14. Pacientes nos estágios IIIa ou IIIb tiveram sobrevida de $73 \%$, inferior à encontrada por Vázquez et al. 13. Para o estágio IV a sobrevida foi de $57 \%$ superior à apresentada por Vázquez et al. 13 .

Neste estudo, embora somente $19,8 \%$ das pacientes se encontrassem no estadiamento I (Tabela 2), a sobrevida estimada foi de $87,7 \%$, provavelmente por fatores considerados de bom prognóstico como: presença de receptor estrogênio positivo em 119 (47,2\%) pacientes; mulheres na pós-menopausa (55\% das pacientes tinham idade superior a 50 anos); ausência de comprometimento linfonodal em $60,3 \%$ das pacientes 15 . Outro fator importante na sobrevida é o tratamento associado à cirurgia como a radioterapia pós-operatória que foi realizada em $60,7 \%$ das pacientes e a quimioterapia em $64 \% 16,17$.

A idade média das pacientes foi 54 anos não sendo um fator prognóstico associado à sobrevida, discordando de outros estudos 11,13,18,19.

A prevalência de carcinoma ductal invasivo foi de $73,4 \%$, apresentando comportamento similar a outros estudos 11,13,17,18,19.

O grau histológico não mostrou significância na avaliação da curva de sobrevida para os diferentes graus considerados, corroborando com as controvérsias encontradas na literatura, que relata dificuldades de reprodutibilidade entre diferentes patologistas e entre diferentes serviços, devido à subjetividade de sua determinação 11,13,17.

O número de linfonodos ressecados mostrou significância estatística na avaliação da sobrevida global, muito provavelmente por possibilitar a detecção da doença micrometastática com maior chance, haja vista que o aumento da acurácia do estadiamento axilar histopatológico se reflete na indicação da terapia adjuvante que melhora o prognóstico da paciente com doença metastática linfonodal 17. Eisenberg 11 não encontrou associação significativa para essa variável, destacando que não existe consenso na literatura quanto a este ser fator prognóstico.

Na abordagem cirúrgica do câncer de mama, a dissecção axilar é um dos fatores mais 


\section{Figura 2}

Curvas de sobrevida estratificadas.

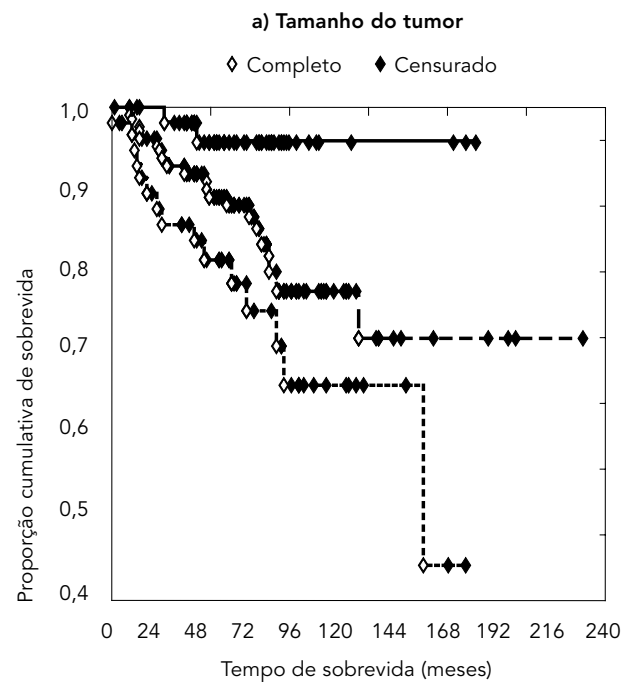

c) Linfonodos regionais comprometidos $\diamond$ Completo Censurado

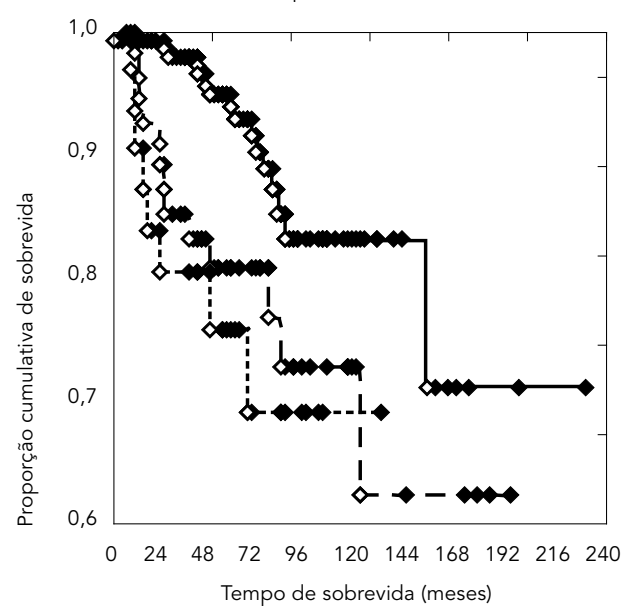

e) c-erbB-2

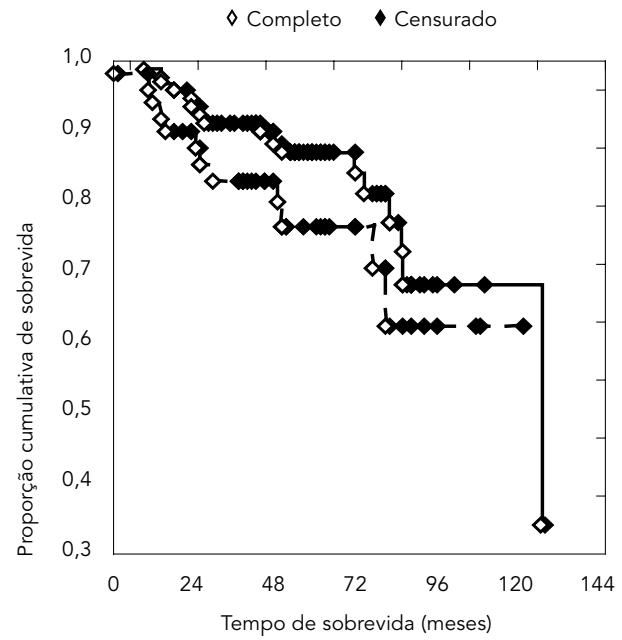

b) Número de linfonodos ressecados

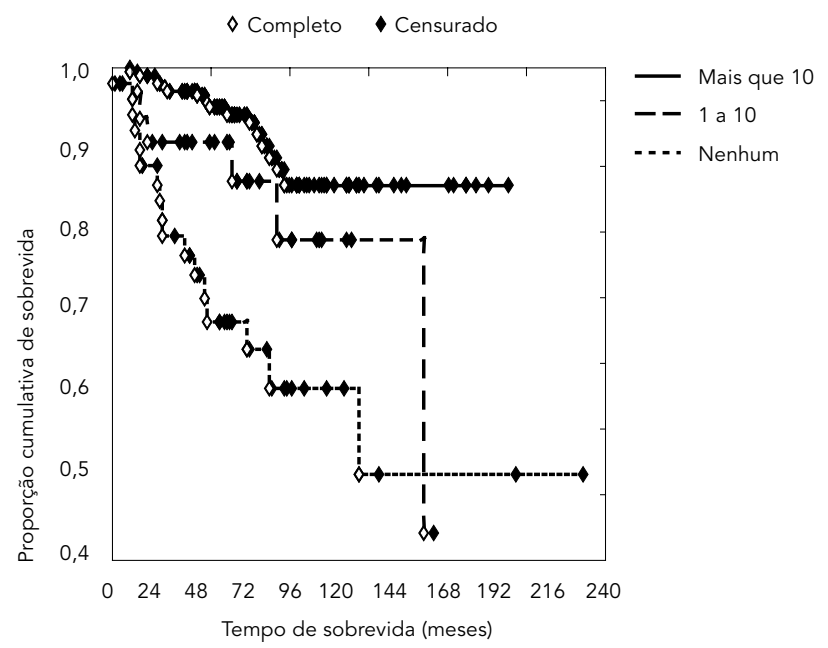

- 2 a $5 \mathrm{~cm}$

-... Maior que $5 \mathrm{~cm}$

d) Receptor de estrógeno

$\diamond$ Completo Censurado

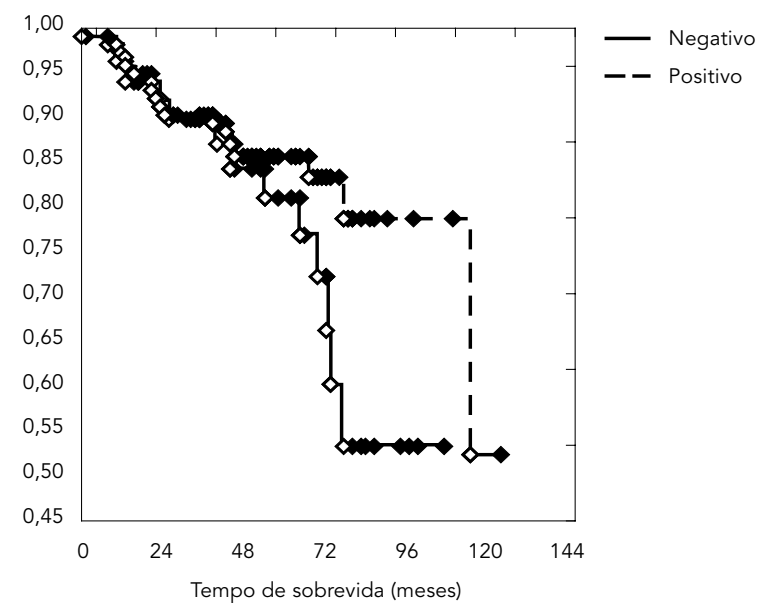


controversos na atualidade, sendo que a maioria das pacientes submetidas à linfadenectomia axilar apresenta elevada morbidade e complicações como: dor axilar persistente, parestesias e alteração da motibilidade, assim como presença de edema no membro superior onde foi realizada a linfadenectomia axilar 20 . A biópsia de linfonodo sentinela está emergindo como um método de amostragem axilar seletiva, minimamente invasivo e altamente sensível para identificação de metástases com acurácia de $96 \%$, sensibilidade de $91 \%$ e especificidade de $100 \% 20$. Um estudo randomizado, conduzido por Veronesi et al. 20, demonstrou que as pacientes submetidas à biópsia de linfonodo sentinela tiveram menor taxa de complicações e melhor mobilidade do que as pacientes submetidas à linfadenectomia axilar completa. Um dos objetivos principais no manejo da paciente com câncer de mama inicial é identificar aquelas com maior probabilidade de doença metastática microscópica 17.

O tamanho do tumor, neste estudo, esteve associado com a sobrevida, sendo menor para as pacientes com tumores de tamanho maior, concordando com a literatura 11,12,19,21.

A presença dos receptores de c-erbB-2 foi considerado um fator de mau prognóstico, já que a presença deste receptor estimula a proliferação celular, estando de acordo com estudos já publicados 11,16,17. Silva et al. 22, em um estudo com 61 pacientes com câncer não metastático, diagnosticado em Goiás, não encontrou associação significativa entre os marcadores p53 e c-erbB-2 com a sobrevida das pacientes.

Na determinação dos programas terapêuticos para paciente com câncer de mama, o conhecimento dos fatores prognósticos é de fundamental importância, visto que permite a aplicação de diferentes modalidades terapêuticas utilizadas no câncer de mama, com intensidade e efetividades adequadas e individualizadas 17 .

No modelo de Cox multivariável ajustado, os fatores prognósticos, significativamente associados à sobrevida foram: tamanho do tumor, comprometimento dos linfonodos, número de linfonodos ressecados e marcadores tumorais (receptor de estrógeno e c-erbB-2), sendo esses fatores citados na literatura 17 . Eisenberg 11 construiu três modelos, encontrando como fatores prognósticos associados à sobrevida: idade, tratamento, tamanho do tumor, comprometimento da pele, grau histológico, invasão vascular e MIB-1. Vázquez et al. 13 encontraram um modelo para a sobrevida onde os fatores prognósticos associados foram: estadiamento, número de gânglios, grau histológico e idade.
Resultados de ensaios clínicos randomizados demonstraram que a cirurgia conservadora associada à radioterapia complementar apresentou taxa de controle local, intervalo livre de doença e sobrevida global semelhante às obtidas pela de cirurgia radical 9,10. Dessa forma, a abordagem conservadora vem sendo preconizada para o manejo cirúrgico do câncer de mama em estágios iniciais, o que proporciona à paciente melhor adequação psíquica e física ao tratamento 9,10 . O tratamento cirúrgico do tipo mastectomia total (Patey) foi realizado em 60,3\% das pacientes, apresentando um fator prognóstico positivo $(\mathrm{HR}=0,46)$ em relação aos outros procedimentos cirúrgicos. Em estudo retrospectivo em câncer de mama localmente avançado (IIIb), realizado no México, Vargas 23 encontrou uma sobrevida menor nas pacientes que não realizaram cirurgia.

Este estudo verificou que $75,8 \%$ das pacientes, quando diagnosticadas, tinham o tumor maior que $2 \mathrm{~cm}$, o que significa diagnóstico em fase mais tardia da doença - estadiamento clínico II. Deve-se considerar que, muito provavelmente, uma das causas no retardo de diagnóstico é a falta de uma política consistente de controle da doença através do diagnóstico precoce pela mamografia de rastreamento. Estudo de Tabar et al. 24 mostrou que há uma redução de $44 \%$ de mortalidade por câncer de mama em mulheres rastreadas por meio de mamografia entre 40-69 anos em países desenvolvidos. Entretanto, o Sistema Único de Saúde (SUS), responsável pela assistência médica de $70 \%$ dos brasileiros, realizou, em 1999, mamografias somente em $8 \%$ das mulheres maiores de 40 anos. Além de insuficientes em número, os mamógrafos existentes no Brasil encontram-se mal distribuídos, estando em sua grande maioria instalados em clínicas radiológicas privadas 25 .

Este estudo apresentou algumas limitações por tratar-se de um estudo retrospectivo de base hospitalar, no qual existe o risco da perda de dados em prontuários, bem como do seguimento de pacientes. Por esse motivo, não encontrou-se no prontuário das pacientes importantes fatores prognósticos como: angiogênese, catepsina D e DNA. Entretanto, deve-se salientar sua importância, pois possibilita a obtenção de informações úteis à administração do hospital e aos pesquisadores interessados nos resultados do tratamento nos diferentes grupos, além de identificar fatores preditores da evolução e da provável recidiva da doença. Os autores identificaram elementos objetivos na análise dos resultados do tratamento realizado pelo SUS em um centro de referência e ensino, como se caracteriza o HUSM, o qual segue as normas 
preconizadas do consenso brasileiro para controle do câncer de mama, que recomenda uma equipe interdisciplinar que deve ser composta por: enfermeiro, psicólogo, fisioterapeuta, terapeuta ocupacional, assistente social e nutricionista junto a pacientes e familiares 26 .

O consenso brasileiro para controle do câncer de mama propõe medidas de capacitação dos profissionais, assim como, recomenda ao SUS estimular medidas educativas nos meios de comunicação e implantar o rastreamento mamográfico no país 26 . Dentre as medidas educativas devemos salientar que o tabagismo, a obesidade, a história familiar, as mutações genéticas, o uso de terapia de reposição hormonal e de anticoncepcionais, bem como o uso de antibióticos, são considerados prováveis fatores de risco para o desenvolvimento dessa doença $4,27,28$.

\section{Resumo}

O objetivo deste estudo retrospectivo de base hospitalar foi descrever as condições de saúde e estimar a sobrevida de 252 pacientes, com diagnóstico prévio de câncer de mama, tratadas e acompanhadas no Ambulatório de Mastologia do Hospital Universitário de Santa Maria, Rio Grande do Sul, Brasil, no período de 1980 a 2000. Para a análise estatística foram utilizados o estimador de Kaplan-Meier e o modelo de Cox. A idade média das pacientes foi 54 anos, sendo que 73,4\% apresentaram diagnóstico histológico de carcinoma ductal invasor, 63,9\% não apresentaram comprometimento dos linfonodos regionais e 57,6\% encontravamse no estadiamento clínico II. Ao final do estudo, 64,7\% das mulheres estavam vivas e sem câncer de mama, $e$ $5,1 \%$ morreram por outra causa. A sobrevida estimada em 5 anos, foi de 87,7\%, sendo os fatores prognósticos associados: tamanho do tumor $(R R=12,03 ;>5 \mathrm{~cm})$, comprometimento $(R R=3,08 ; N 1)$ e número de linfonodos ressecados $(R R=4,66$; Nenhum), receptor de estrógeno $(R R=0,34)$ e c-erbB-2 $(R R=2,51)$. Com base nos achados deste estudo, destaca-se a importância de ações intensivas de orientação para implementar o rastreamento do câncer de mama, levando a diagnósticos em estádios precoces dessa neoplasia.

Análise de Sobrevida; Neoplasias Mamárias; Assistência à Saúde; Saúde da Mulher
A taxa de sobrevida encontrada pode ser utilizada para estimar a eficiência global do sistema de saúde, que depende não só da qualidade do cuidado prestado, mas também da acessibilidade ao sistema de saúde e, conseqüentemente, estimar a probabilidade de um diagnóstico e tratamento precoces 29.

O câncer de mama vem sendo considerado uma epidemia nas últimas décadas 1 . Infelizmente, no Brasil, o aumento da incidência tem sido acompanhado pelo aumento da mortalidade por esse câncer, o que pode ser atribuído ao diagnóstico tardio e à falta de implementação da terapêutica adequada 27. Dessa forma, é imprescindível que os órgãos competentes de saúde promovam campanhas intensivas de educação e orientação, bem como o rastreamento do câncer de mama, para que possam ter diagnósticos em estádios mais precoces dessa neoplasia.

\section{Colaboradores}

A. B. Moraes e R. R. Zanini participaram de todas as etapas do estudo e da elaboração do artigo. M. S. Turchiello participou na etapa de amostragem, organização e construção do banco de dados. J. Riboldi colaborou na orientação, revisão e redação do artigo. L. R. Medeiros colaborou na revisão e redação do artigo.

\section{Agradecimentos}

Os autores deste trabalho agradecem ao Hospital Universitário de Santa Maria pela disponibilidade dos dados e à Fundação de Amparo à Pesquisa do Estado do Rio Grande do Sul, pelo apoio financeiro para a execução deste projeto (processo n. 01513266). 


\section{Referências}

1. Sasco AJ. Breast cancer and environment. Horm Res 2003; 60 Suppl 3:50.

2. Instituto Nacional de Câncer. Câncer de mama. http://www.inca.gov.br/conteudo_view.asp?id=3 36 (acessado em 05/Mai/2005).

3. E-Cancer: Informações para uma vida melhor. Câncer de mama. http://andre.sasse.com/mama. htm (acessado em 05/Ago/2004).

4. Pedersen L, Gunnarsdottir KA, Rasmussen BB, Moeller S, Lanng C. The prognostic influence of multifocality in breast cancer patients. Breast 2004; 13:188-93.

5. Fleming ID, Cooper JS, Henson DE. AJCC cancer staging manual. Philadelphia: Lippincott-Raven; 1997.

6. Elston CW, Ellis IO. Pathological prognostic factors in breast cancer. The value of histological grade in breast cancer: experience from a large study with long-term follow-up. Histopathology 1991; 19:403-10.

7. Cox DR, Oakes D. Analysis of survival data. London: Chapman and Hall; 1984.

8. Hosmer Jr. DW, Lemeshow S. Applied survival analysis: regression modeling of time to event data. New York: John Wiley \& Sons; 1998.

9. Veronesi U, Cascinelli N, Mariani L, Greco M, Saccozzi R, Luini A, et al. Twenty-year follow-up of a randomized study comparing breast - conserving surgery with radical mastectomy for early breast cancer. N Engl J Med 2002; 347:1227-32.

10. Fischer B, Anderson S, Bryant J, Margolese RG, Deutsch M, Fischer ER, et al. Twenty-year followup of a randomized trial comparing total mastectomy, lumpectomy, and lumpectomy plus irradiation for the treatment of invasive breast cancer. N Engl J Med 2002; 347:1233-41.

11. Einsenberg ALM. Sobrevida de cinco anos para pacientes com carcinoma ductal infiltrante de mama sem comprometimento de linfonodos axilares: coorte hospitalar, 1992-1996 [Tese de Doutorado]. Rio de Janeiro: Escola Nacional de Saúde Pública, Fundação Oswaldo Cruz; 2001.

12. Moreno-de-Miguel LF, Pérez-Braojo I, SánchezVarela I, Rodríguez-Díaz R. Cirugía conservadora más radioterapia en el cáncer temprano de mama en el INOR. Rev Cuba Oncol 1998; 14:143-8.

13. Vázquez T, Krygier G, Barrios E, Cataldi S, Vázquez A, Alonso R, et al. Análisis de sobrevida de una población con cáncer de mama y su relación con factores pronósticos: estudio de 1.311 pacientes seguidas durante 230 meses. Rev Med Urug 2005; 21:107-21.

14. Mora-Díaz I, Sánchez-Redonet E. Estado actual de las pacientes con cáncer de mama em estadio I y II. Rev Cuba Obstet Ginecol 2004; 30(1). http:// scielo.sld.cu/scielo.php?script=sci_arttext\&pid=S 0138-600X2004000100009\&lng=es\&nrm=iso (acessado em 08/Dez/2005).
15. Kroman N, Jensen MB, Wohlfahrt J, Mouridsen HT, Andersen PK, Melbye M. Factors influencing the effect of age on prognosis in breast cancer: population based study. BMJ 2000; 320:474-8.

16. Tutt A, Ross G. Commentary: much still to learn about relations between tumour biology, prognosis, and treatment outcome in early breast cancer. BMJ 2000; 320:478-9.

17. Abreu E, Koifman S. Fatores prognósticos no câncer da mama feminina. Rev Bras Cancerol 2002; 48:113-31.

18. Brito C. Avaliação do tratamento à paciente com câncer de mama nas unidades oncológicas do Sistema Único de Saúde no Estado do Rio de Janeiro [Dissertação de Mestrado]. Rio de Janeiro: Escola Nacional de Saúde Pública, Fundação Oswaldo Cruz; 2004.

19. Pereira WMM. Mortalidade e sobrevida por câncer de mama no Estado do Pará [Dissertação de Mestrado]. Rio de Janeiro: Escola Nacional de Saúde Pública, Fundação Oswaldo Cruz; 2001.

20. Veronesi U, Paganelli G, Viale G, Luini A, Zurrida $S$, Galimberti V, et al. A randomized comparison of sentinel-node biopsy with routine axillary dissection in breast cancer. N Engl J Med 2003; 349:546-53.

21. Korzeniowski S, Dyba T, Skolyszewski J. Classical prognostic factors for survival and locoregional control in breast cancer patients treated with radical mastectomy alone. Acta Oncol 1994; 33:75965.

22. Silva DM, Saddi VA, Momotuk EG. Marcadores moleculares associados ao câncer de mama não metastático. Rev Bras Cancerol 2002; 48:39-48.

23. Vargas AN. Mastectomía toilet: manejo paliativo en mujeres con cáncer de mama avanzado. Ginecol Obstet Mex 2002; 70:392-7.

24. Tabar L, Yen MF, Vitak B, Chen HHT, Smith RA, Duffy SW. Mammography service screening and mortality in breast cancer patients: 20 years follow-up before and after introduction of screening. Lancet 2002; 361:1405-10.

25. Koch HA, Peixoto JE, Neves ALE. Análise da infraestrutura para a mamografia. Radiol Bras 2000; 33:23-9.

26. Ministério da Saúde. Controle do câncer de mama-documento de consenso. Rev Bras Cancerol 2004; 50:77-90.

27. ESHRE Capri Workshop Group. Hormones and breast cancer. Hum Reprod Update 2004; 10:281-93.

28. Boyle P, Leon ME, Maiosonneuve P, Autier P. Cancer control in women. Update 2003. Int J Gynaecol Obstet 2003; 83 Suppl 1:179-202.

29. Bustamente-Teixeira MT, Faerstein E, Latorre MR. Técnicas de análise de sobrevida. Cad Saúde Publica 2002; 18:579-94.

Recebido em 25/Jul/2005

Versão final reapresentada em 06/Jan/2006 Aprovado em 17/Mar/2006 